\title{
Spinal Injuries Caused By The Acceleration Of Ejection
}

\author{
ME Lewis
}

\begin{abstract}
The speed and altititude at which modern military aircraft operate are such that escape can only be achieved by some means of forcibly propelling the aircrew clear of the aircraft. The most common method of doing this is by use of an ejection seat. The use of such seats, whilst generally life saving, exposes aircrew to forces that may be at the limits of human tolerance. Each phase of the ejection sequence is associated with characteristic injury patterns and of particular concern is the occurrence of spinal compression fractures, which are caused by the upward acceleration of the ejection seat. Thorough investigation of aircrew who eject is necessary and magnetic resonance imaging of the spines of these aircrew is now becoming mandatory. Aircrew who sustain stable anterior wedge compression fractures usually require no invasive treatment, but are prevented from flying aircraft fitted with ejection seats for 3-4 months.
\end{abstract}

\section{Introduction}

The need for ejection systems, to provide a means of actively removing the aircrew from their aircraft in an attempt to overcome the difficulties associated with manual escape, was first considered in Sweden and Germany and then later in the UK. The increase in speed of military aircraft developed during the 1939-1945 war resulted in manual escape becoming an increasingly difficult task. The high speed of the aircraft subjected escapees to severe airblast loads, which could physically prevent the aircrew from climbing out of the cockpit or could increase the possibility of failure to survive the escape from subsequent contact with the aircraft structure. The development of the ejection seat could overcome these problems. Nevertheless, ejection is still not without its dangers, and the development of escape systems has brought additional hazards to which pilots bailing out from aircraft were not exposed.

The scope of this paper is to outline the ejection sequence, to discuss the accelerations imposed on the aircrew and to highlight the spinal injuries which may result. The paper describes each phase of the ejection sequence: ejection initiation, escape pathway clearance, initial seat acceleration, exposure to windblast, man/seat separation, main parachute canopy deployment and parachute landing, and relates these phases with the injuries the ejectees may sustain.

\section{Ejection Sequence}

The ejection sequence varies slightly with aircraft and ejection seat types; however, all assisted escape systems have broadly similar modes of operation. The emergency escape systems fitted in RAF fast jet aircraft are initiated by pulling a seat pan firing handle. This fires the initiation cartridge and gas is piped around the seat to:

- The harness power retraction unit, which pulls the seat occupant's shoulders and upper torso backward so as to improve the aircrew's posture and spinal alignment.

- The ejection gun sear withdrawal unit, which rotates the cross shaft assembly and fires the primary cartridge of the ejection gun.

- The command ejection selector valve, which fires the command ejection cartridge (if fitted) and hence, initiates the ejection sequence of the second seat of a twin seat aircraft.

- The Miniature Detonating Cord (MDC) or canopy jettison firing unit.

The gases from the ejection gun primary cartridge initiate upward seat movement, release the locking plunger from the top latch and thus unlock the sear from the aircraft structure. As the seat accelerates up the guide rails the following events occur:

- The ejection gun secondary cartridges fire in turn to maintain the upward acceleration of the seat.

- The static trip rods withdraw the sears from the drogue gun and the barostatic time release unit; the command ejection system disconnects as does the aircraft portion of the personal equipment connector and the main oxygen system.

- The emergency oxygen supply is selected and the regulator is set to $100 \% 0_{2}$

- The leg restraint lines, and arm restraint lines, if fitted, are pulled downwards through the snubber units to restrain the occupant's limbs.

- The rocket motor initiator cartridge is fired by the pull of a static cable.

Rocket motor initiation is timed to occur immediately prior to gun separation, so that the seat continues to accelerate smoothly 
away from the aircraft. As the seat separates from the aircraft it will still have a high forward speed which must be slowed down before the main parachute can be deployed safely. Furthermore, the seat has poor aerodynamic properties which could potentially cause it to spin and tumble, and as a consequence it needs to be stabilised on exposure to the windblast forces. The drogue parachute system stabilises and decelerates the seat to a safe speed for main parachute canopy deployment. The drogue parachute is extracted from the seat by the drogue gun bullet which is timed to fire after the seat has cleared the cockpit structures. Following a possible short time delay (if the ejection occurs above the set Barostatic Time Release Unit height, usually $10,000 \mathrm{ft}$ ) the locks securing the occupant's harness to the ejection seat are released and the pull of the drogue is transferred from the ejection seat to the parachute withdrawal line, producing main parachute extraction. As the man separates from the seat, the pull of the drogue parachute continues on the main parachute canopy to inflate it fully. The aircrew then descends to the ground beneath the main parachute canopy.

\section{Ejection Injury}

The use of ejection seats to allow aircrew to escape from aircraft is generally lifesaving. However, their use exposes aircrew to forces that may be at the limits of human tolerance.

The ejection sequence occurs extremely rapidly with the complete sequence, from initiation to being suspended on a fully deployed canopy taking approximately $2.5 \mathrm{~s}$. Each phase of the ejection sequence can result in aircrew sustaining specific injuries and these injuries are summarised in Table 1.

Table 1. Typical injuries which may be sustained during the phases of the ejection sequence on ejecting from a fast jet aircraft.

\begin{tabular}{|c|c|}
\hline Phase of Ejection Sequence & $\begin{array}{l}\text { Possible Injuries Occurring During } \\
\text { Each Phase of the Ejection Sequence }\end{array}$ \\
\hline Escape pathway clearance & $\begin{array}{l}\text { MDC splatter burns. } \\
\text { Canopy jettison rocker motor flash burns. } \\
\text { Cervical spine, head, shoulder, limb injury } \\
\text { resulting from through canopy ejection. }\end{array}$ \\
\hline Ejection gun firing and rocket motor burn & $\begin{array}{l}\text { Spinal compression fractures. } \\
\text { Femoral fracture from contact with seat pan. }\end{array}$ \\
\hline Windblast & Windblast flail injuries \\
\hline Drogue parachute deployment & $\begin{array}{l}\text { Spinal injury from drogue parachute } \\
\text { opening shock loads. }\end{array}$ \\
\hline Main parachute canopy deployment & $\begin{array}{l}\text { Spinal injury from main parachute opening } \\
\text { shock loads. } \\
\text { Head and cervical spine injury from helmet } \\
\text { and parachute riser interaction. }\end{array}$ \\
\hline Parachute landing & $\begin{array}{l}\text { Lower limb fractures. } \\
\text { Spinal injuries. }\end{array}$ \\
\hline
\end{tabular}

\section{Spinal Injury}

Emergency escape from combat aircraft exposes aircrew to vertical accelerations that approach, and may exceed, vertebral compression failure limits. The firing of the ejection gun applies an acceleration that is primarily in the upward $(\mathrm{Gz})$ direction and the duration of thrust is limited to the time the seat is attached to the ejection gun, unless the seat is fitted with a rocket pack. The objective is to attain the highest possible velocity in this time and, hence large accelerations are applied very suddenly. The problem of vertebral compression fractures was first observed on the early German ejection seats (1). Prototype versions of these seats produced peak accelerations of $12 \mathrm{G}$ and rates of rise (jolt) of $1100 \mathrm{Gs}^{-1}$. Although the value of $12 \mathrm{G}$ is known to be comfortably within the limits of human tolerance, the figure of $1100 \mathrm{Gs}^{-1}$ would now be regarded as extremely hazardous.

In the UK, work was directed towards the systematic investigation of the physiological limits of jolt and peak acceleration and much effort was expended using different propulsive charges and types of ejection guns to produce accelerations that were subjectively tolerable to humans (2). The outcome of this work was the setting of limiting values of peak acceleration at $25 \mathrm{G}$ and jolt to $300 \mathrm{Gs}^{-1}$.

Ejection seats manufactured by the Martin Baker Aircraft Company began to be incorporated into RAF combat aircraft during the 1940s, but it was not until the late 1950s that sufficient experience in their use had occurred to permit an analysis of the injuries sustained. The earliest ejection seats had ejection gun velocities of $53 \mathrm{ft} \mathrm{s}^{-1}$ and 60 $\mathrm{ft} \mathrm{s}^{-1}$, but in later seats the gun velocity was increased to $80 \mathrm{ft} \mathrm{s}^{-1}$. An $80 \mathrm{ft} \mathrm{s}^{-1}$ velocity ejection gun enabled aircrew to eject safely at zero altitude, as sufficient height was gained to allow main parachute canopy deployment. Furthermore, the increase in gun velocity permitted the aircrew to eject at a high air speed so that clearance of the tail fin was achieved. Full zero altitude, zero forward speed ejection, however, was not yet possible as some forward speed was required to give sufficient airflow so that the main parachute canopy could deploy. Fryer (3) analysed $\mathrm{RAF}$ and $\mathrm{RN}$ ejections and showed there was an increase in vertebral fracture rates from $10 \%$ to $35 \%$ associated with the increase in the acceleration of the $80 \mathrm{ft} \mathrm{s}^{-1}$ ejection gun.

During the 1960s considerable technical development of the ejection seat took place, which was driven by the dual need to automate the ejection sequence and to increase the safe ejection envelope. This lead to the introduction of the rocket assisted ejection seat, which permitted the down rating of the ejection gun from $80 \mathrm{ft} \mathrm{s}^{-1}$ to 64 $\mathrm{ft} \mathrm{s}^{-1}$. Down rating the ejection gun to $64 \mathrm{ft} \mathrm{s}^{-1}$ reduced the acceleration acting on the spine 
and thereby reduced the spinal fracture rate. Moreover, the effect of the rocket motor was to augment the ejection seat thrust so that sufficient height was attained to allow the main parachute to deploy when the ejection occurred at zero altitude and zero forward speed. The thrust characteristics resulted in considerable improvement in both the seat escape envelope and the force time curve of the early part of the ejection sequence. Since Fryer's original analysis was performed numerous other studies have analysed spinal injury rates following ejection and these results have consistently shown a peak incidence of fractures around the $\mathrm{T} 12$ and L1 level $(4,5,6,7)$.

\section{Appearance of Spinal Lesions}

Several types of fractures may occur following ejection, but by far the most common is the anterior wedge compression fracture caused by combined vertical compression and forward flexion of the spine.

An anterior wedge compression fracture is seen radiologically as a reduction in the anterior height of the vertebral body. One or several vertebrae may be affected and the same features may be seen in all the affected vertebrae. Lateral $\mathrm{X}$-ray views are the most appropriate and will define the degree of compression (Figure 1). In most cases following ejection the loss of vertebral height is slight, but on occasions the height may be reduced by two thirds. Frontal X-rays can show widening of the vertebral body and occasionally, an element of lateral compression may be present with the



Fig 1. Radiograph of an anterior wedge compression fracture of L3 vertebrae. anterior posterior X-rays showing asymmetry of the vertebral height in the frontal plane. In a number of cases the anterior superior corner is torn away and the line of fracture is visible as an irregular or serrated edge with the anterior border of the vertebral body deformed to an obtuse angle. Compression fractures are usually accompanied by disruption of the anterior part of the end plate and in the majority of cases only the upper vertebral surface is affected.

It is believed that the position of the pilot during ejection is a critical factor in causing spinal fractures. An inappropriate sitting position may lead to relative weakness in the vertebral column and can give rise to injury when the acceleration would otherwise be tolerable. The factors that modify the position of the pilot are numerous. The attitude of the aircraft at the time of the ejection is important as it alters the relationship between the seat and the pilot. Thus, in a nose-down ejection the pelvis may separate from the seat even if the harness is properly tightened. Similarly in ejections from a steeply banked aircraft there is lateral flexion which is all the greater if the harness is poorly adjusted. A very slack harness gives greater freedom of movement of the trunk, which bends considerably during ejection. In the earlier ejection seats fitted with a face blind, forward flexion of the trunk was limited by retention of the head behind the face blind.

The major component of the ejection acceleration is in the long axis of the spine; however, significant flexion forces are present and arise because the line of seat thrust does not coincide with the long axis of the spine. The included angle is the angle between the axis of the spine and the line of thrust and large values favour the development of fractures of the vertebral column by hyperflexion. Even when sitting in the correct ejection posture the geometry of the seat structure prevents the spine from aligning with the axis of thrust and when the included angle is large it will have the same effect as exaggerated flexion of the trunk.

\section{Through Canopy Ejection and Spinal Injuries}

Ejection through an intact canopy has over recent years been phased out as the primary method of ejection path clearance with ejection occurring either through a shattered cockpit canopy, by the action of fragmentation devices, or by canopy jettison. However, ejection through an intact cockpit canopy can occur if there has been a failure of the primary canopy clearance mechanism. If an ejection occurs through canopy, three new hazards may arise:

- Modification of the acceleration profile for the seat and of the seat occupant causes 


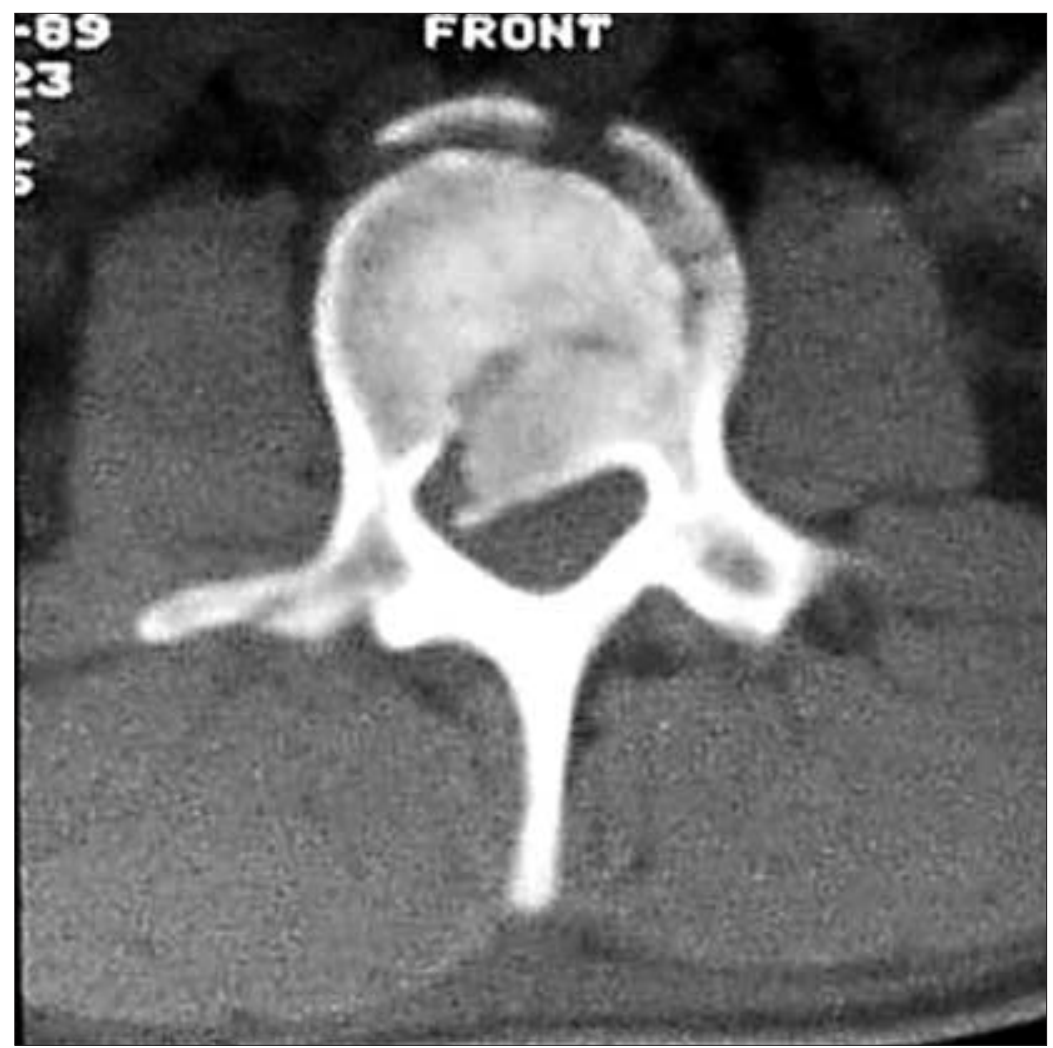

Fig 2. Burst fracture of vertebral body caused by through canopy ejection.

greater accelerations at the level of the seat and of the body segments represented by the pelvis thorax and head. This produces greater compression of the vertebrae.

- Impact between the canopy and the head, shoulders and knees.

- Tearing of the protective clothing, damage to survival equipment and laceration to underlying tissue may be produced by fragments of transparency which have pierced various layers of clothing (8).

At the onset, the acceleration of the seat is moderately high and it then decays rapidly as the canopy breakers come into contact with the canopy. The upward movement of the seat is momentarily retarded. During this time the acceleration at the pelvis of the seat occupant progressively increases and the occupant continues on its upward path, despite the retardation of the seat, as if it had parted company with the seat. When the canopy breaks, the seat accelerates again, at first slowly during the disruption of the canopy, then more rapidly for a very short period when there is no longer an obstacle to its passage. Finally the seat catches up with the occupant producing a secondary acceleration of the occupant and a deceleration of the seat. Thereafter, the acceleration profile of the seat and the occupant follow almost parallel paths. The alteration in the acceleration profile from through canopy ejection can cause an increase in the forces acting on the spine and as a result more severe injuries, such as burst fractures, may be sustained. Furthermore, if the aircrew is sitting high, with the seat pan motored up, there is a potential for the crown of the head to be above the top of the headbox and canopy breakers. A through canopy ejection in such cases would result in the head striking the canopy first. Forced forward flexion of the head may then occur and serious cervical spine injuries can result. Furthermore, sternum fractures may occur from forcible contact of the chest, either by the chin as the forward flexed head comes in to contact with the transparency, or when the chest is struck by a piece of detached canopy.

The development of stronger and thicker canopy transparencies, in particular to protect the aircrew from birdstrike injury, has lead to the use of thicker transparencies using stretched acrylic instead of the earlier Plexiglas and cast acrylic. The fragmentation characteristics of the transparency materials differ significantly, and instead of a large number of small blunt edged fragments resulting from the break up of cast acrylic canopies, stretched acrylic breaks into a small number of larger pieces with razor sharp edges capable of inflicting serious injury to aircrew and damage to aircrew equipment assemblies. The physical characteristics of stretched acrylic alter with temperature. In response to an increase in temperature stretched acrylic becomes more malleable and resists penetration as it stretches and balloons over the emerging seat (9). The higher the airspeed then the higher the temperature of the canopy and the higher the resistance to penetration. In comparison with early experience of injuries received on ejection through cast acrylic canopies, penetration through stretched acrylic will cause more severe acceleration injuries and injuries arising from contact with heavier, larger and sharper canopy fragments.

\section{Investigation and Management of Ejection Spinal Injuries}

The recent experience of the RAF has demonstrated that following an ejection, spinal injuries may be ill defined on plain Xrays, despite the presence of minor symptoms. For this reason it is important that all aircrew who eject from an aircraft, and those who have been exposed to high vertical decelerations, are investigated fully. It is now the requirement that all UK military personnel who eject from an aircraft have a magnetic resonance (MR) scan of their spines performed prior to returning to flying fast jet aircraft. This investigation will not only confirm the presence of any fractures, but may also identify soft tissue injuries. Furthermore, the ejectee should also have a neurological assessment by a consultant neurologist or neurosurgeon at an approved specialist centre. Ideally, a whole body isotope bone scan 3-14 days post ejection should be performed, but a MR scan of the brain should be limited to those who have had or suspected to have had a head impact.

If stable anterior wedge compression 
fractures have been sustained, it is usual that aircrew are prevented from flying fast jet aircraft for approximately 3-4 months. This lay-off period, therefore, will enable the injury to heal. Should the aircrew return to fast jet flying before the compression fracture has healed, and an emergency arises again forcing the aircrew to eject, then the resulting acceleration induced injury could potentially be severe. If the aircrew had sustained an unstable spinal fracture (Figure 2), which has necessitated internal fixation and who have metal fixation in place, then the aircrew are not to fly ejection seat fitted aircraft. The effects of the metal fixation on spine when subjected to the accelerations of ejection are unknown and the pathway of the forces through the spine cannot be easily predicated. Consequently, aircrew may expose themselves to potentially crippling forces should they eject with metal fixation in situ. Unstable compression fractures are, however, a rare occurrence following ejection. Nevertheless, a RAF pilot has returned to flying ejection seat aircraft after sustaining an unstable spinal fracture which necessitated surgical fixation, but the return to fast jet flying occurred following a second surgical operation, at eight months post ejection, to remove the metal fixation.

\section{Summary}

Escaping from an aircraft by means of an ejection seat is without question manifestly dangerous and can expose the aircrew to injurious levels of spinal accelerations. Even so, ejection seats have saved many thousands of lives and though the potential for injury is ever present, technical improvements are ongoing in an attempt to reduce the spinal injury threat for ejectees. Aircraft manufacturers and operational requirements are dictating that escape systems should operate safely under increasingly hostile environments and at great speed and at high or low altitude. Therefore, constant programs of improvements are required, not only for escape systems currently in operational use, but also for future escape systems, to ensure aircrew eject safely and injuries are minimised in order to permit aircrew to return to operational flying.

\section{References}

1. Goertz A. Limits and special problems in the use of ejector seats - translation of a dissertation for the degree of Doctor of Engineering, University of Rostock. Defence Research Information Centre 1954; DRIC-T-7684.

2. Stewart WK. Ejection of pilots from aircraft - a review of the applied physiology. Flying Personnel Research Committee 1946; Report Number 671.

3. Fryer DI. Operational experience with British ejection seats. Flying Personnel Research Committee 1961; Report Number 1166.

4. Anton DJ. The Incidence of Spinal Fractures on RAF Ejections 1968-1983, RAF Institute of Aviation Medicine Aircrew Equipment Group 1986; Report No 529.

5. Newman DG. The ejection experience of the Royal Australian Air Force: 1951-92. Aviat Space Environ Med 1995; 66: 45-49.

6. Sandstedt P. Experience of rocket seat ejections in the Swedish Air Force: 1967-1987. Aviat Space Environ Med 1989; 60: 367-73.

7. Werner U. Ejection associated injuries within the German Air Force from 1981-1997. Aviat Space Environ Med 1999; 70: 1230-4.

8. Chiou WY, Ho BL, Kellogg DL. Hazard potential of ejection with canopy fragments. Aviat Space Environ Med 1993; 64: 9-13.

9. Langdon G. Ejection of the type $10 \mathrm{~A}$ seat through an unbroken $9 \mathrm{~mm}$ aircraft canopy. Aeroplane and Armament Experimental Establishment 1974; Note No 3176. 